\title{
Characters Association and Path Analysis among CMS and SI Based Cabbage Hybrids under Mid Hill Conditions of Himachal Pradesh, India
}

\author{
Manmeet Kaur, Sanjay Chadha*, Nitish Kumar, Nidhi Sehgal and Sangeeta Kanwar \\ Department of Vegetable Science and Floriculture, College of Agriculture, CSK Himachal \\ Pradesh Krishi Vishvavidyalaya, Palampur- 176062, India \\ *Corresponding author
}

\section{A B S T R A C T}

\begin{tabular}{|l|}
\hline K e y w o r d s \\
Cabbage, Brassica \\
oleracea var. \\
capitata L, Hybrids, \\
Correlation, Path \\
analysis
\end{tabular}

\section{Keywords}

Cabbage, Brassica oleracea var. capitata L, Hybrids, analysis

Article Info

Accepted: Available Online

\section{Introduction}

Cabbage, Brassica oleracea var. capitata L. $(2 \mathrm{n}=2 \mathrm{x}=18)$ member of family Brassicaceae is one of the most important cole-group vegetable crops originated from Brassica oleracea var. oleracea L. (syn. Brassica oleracea var. sylvestris L.) commonly known as wild cabbage through mutation, human selection and adaptation. It is rich source of sulphur containing amino acids, minerals, carotenes, ascorbic acid and anti-carcinogenic property (Kopsell et al., 2004 and Singh et al., 2009). Cabbage is grown throughout the world
A field trial was conducted for evaluation of 38 hybrids of cabbage at the Experimental Farm, Department of Vegetable Science and Floriculture, CSK HPKV, Palampur, (H.P.) during Rabi 2015-16 to generate information on correlation and path analysis. The periment was laid out in a Randomized Block Design (RBD) with three replications. (he plant spread, gross head weight, net head weight, polar diameter, equatorial diameter, marketable heads per plot, ascorbic acid plot at a characters either in combination or alone will result in identifying the genotypes having high yield potential. Estimates of direct effects showed that net weight of head had the highest positive direct effect on marketable head yield per plot followed by polar diameter, compactness of head, ascorbic acid content, number of non-wrapper leaves and TSS at genotypic level. Therefore, net weight of head, polar diameter and compactness of head should be considered as selection criteria for yield improvement in cabbage breeding. 
adaptability under adverse growing conditions. In developed countries more than $90 \%$ cabbage growing area is under hybrid varieties, whereas it is only $31 \%$ in India (Kumar et al., 2013). Thus, it is important to identify the high yielding hybrid(s) with better quality and adaptability. The yield and its component characters are polygenic in nature, hence influenced by the environmental factors. The knowledge of the inter relationship among the various yield components and their direct and indirect effects on yield are the important pre-requisites to bring genetic improvement in cabbage. Path analysis facilitates partitioning of correlation coefficients into direct and indirect effects of various characters on head weight which can prove useful in providing information about improvement of yield or yield related characters. The information regarding the correlation and path coefficient analysis in cabbage is inadequate. Therefore the present study was conceived with objective to estimate the correlation coefficient and path analysis among the different traits of cabbage.

\section{Materials and Methods}

The present investigation was carried out at the Experimental Farm $\left(32^{\circ} 6^{\prime} \mathrm{N}\right.$ latitude, $76^{\circ} 3^{\prime}$ E longitudes and $1290.8 \mathrm{~m}$ altitude) of the Department of Vegetable Science and Floriculture, CSK Himachal Pradesh Krishi Vishvavidyalaya, Palampur, (H.P.) during Rabi, 2015-16 in RBD with three replications at intra and inter-row spacing of $45 \mathrm{~cm}$ each. The experimental materials comprised of 38 cabbage hybrids from public and private sector. The standard cultural practices to raise the crop were followed as per the recommended package of practices for vegetable crops by CSKHPKV, Palampur. Observations were recorded on five competitive randomly marked plants for characters namely plant spread $(\mathrm{cm})$, number of non-wrapper leaves, gross head weight $(\mathrm{g})$, net head weight $(\mathrm{g})$, polar and equatorial diameters of head $(\mathrm{cm})$, days to harvest, head shape index, compactness of head $\left(\mathrm{g} / \mathrm{cm}^{3}\right)$, marketable heads per plot, marketable head yield per plot (kg), ascorbic acid content $(\mathrm{mg} / 100 \mathrm{~g})$ and TSS ( ${ }^{0}$ Brix $)$ in each treatment and each replication. The mean values obtained from the experiment were used for estimating the analysis of variance as suggested by Burton and De Vane (1953). Correlation and path coefficient analysis were calculated as per formulae suggested by AlJibouri et al., (1958) and Dewey and Lu, (1959), respectively. The statistical analysis was carried out by using OPSTAT software.

\section{Results and Discussion}

The effectiveness of any breeding or selection programme depends upon the nature of association between yield and other component characters, as more directly a character is associated with yield in the desirable direction; more will be the success of the selection programme. Genotypic correlation coefficients in general were higher than the phenotypic correlations which revealed that though there is a strong inherent association between various characters, the phenotypic expression of the correlation gets modified under the influence of environment. The perusal of data from Table 1 revealed that at phenotypic level, marketable head yield/plot exhibited significant positive correlation with net head weight (0.940), gross head weight (0.833), equatorial diameter (0.651), marketable heads per plot (0.647), polar diameter (0.538), plant spread (0.427) and compactness of head (0.397). Earlier reports of many research workers have also indicated positive and significant correlation of marketable head yield with net head weight (Singh et al., 2013, Soni et al., 2013 and Kibar et al., 2014), gross head weight (Meena et al., 2009 and Sharma, 2010), equatorial diameter (Meena et al., 2009 and Kibar et al., 2014), 
polar diameter (Soni et al., 2013 and Kibar et al., 2014), plant spread (Soni et al., 2013), compactness of head (Sharma, 2010). Plant spread had positive significant correlation with gross head weight $(0.520)$, net weight of head (0.469), number of non-wrapper leaves (0.359), equatorial diameter (0.464), polar diameter (0.435) and compactness of head (0.194). Singh et al., (2010) had also reported positive correlation of frame spread with polar diameter, equatorial diameter, gross weight and net weight of head.

A significant and positive correlation of number of non-wrapper leaves was recorded only with gross head weight (0.259) at phenotypic level. These results are in consonance with findings of Sharma (2010). Gross head weight had positive and significant correlation with net weight of head (0.891), equatorial diameter (0.672), polar diameter (0.548), marketable heads per plot (0.352) and compactness of head (0.270) and significant negative correlation with days to harvest (0.404) and head shape index (-0.254). These results are in conformity for net head weight (Singh et al., 2010 and Sharma, 2010), equatorial diameter (Singh et al., 2010) and polar diameter (Singh et al., 2010).

Net head weight had significant positive correlation with equatorial diameter (0.635), polar diameter (0.577), marketable heads per plot (0.379), compactness of head (0.315) and ascorbic acid content (0.225) and significant negative correlation with days to harvest (0.490). These results are in agreement with various workers for polar and equatorial diameter (Sharma, 2010 and Kibar et al., 2014). Polar diameter exhibited positive significant correlation with equatorial diameter (0.635), marketable heads per plot (0.256) and head shape index (0.231) and significant negative correlation with days to harvest (-0.254). Similar findings have also been reported for equatorial length (Singh et al., 2010). Equatorial diameter was positively and significantly correlated with marketable heads per plot (0.415) and TSS (0.192). Its association was negative and significant with head shape index $(-0.564)$ and days to harvest (-0.340). Singh et al., (2013) also reported that equatorial diameter had negative but nonsignificant correlation with days to maturity. Head shape index had negative and significant correlation with marketable heads per plot (0.301). Days to harvest had significant negative correlation with marketable heads per plot (-0.483), TSS (-0.220) and compactness of head (-0.199). Compactness of head was significantly and positively correlated with marketable heads per plot (0.285). Sharma (1994) reported compactness of head had positive and significant association with shape of head. Relationship of marketable heads per plot was positive and significant with ascorbic acid content (0.220) and TSS (0.312). Soni et al., (2013) reported positive and significant correlation of ascorbic acid content with plant spread, number of non-wrapper leaves, head weight, head polar diameter and equatorial diameter.

Knowledge of correlation alone, however, is often misleading as the correlation observed may not be true. Two characters may show correlation just because they are correlated with a common third one. In such cases, it becomes necessary to study a method, which takes into account the casual relationship in addition to the degree of relationship. Path coefficient analysis is one such method that takes into account both kind of relationship. The data pertaining to path coefficient analysis (Table 2) revealed that at phenotypic level, net head weight (0.849) and marketable heads per plot (0.327) are the two main characters which showed the maximum direct positive effect on marketable head yield per plot. Whereas, the maximum negative direct effects were observed via gross head weight $(-0.054)$ and head shape index (-0.024). 
Table.1 Estimates of correlation at phenotypic $(P)$ and genotypic $(\mathrm{G})$ levels between different traits of cabbage

\begin{tabular}{|c|c|c|c|c|c|c|c|c|c|c|c|c|c|}
\hline Traits & & $\begin{array}{c}\text { No of } \\
\text { non } \\
\text { wrapper } \\
\text { leaves }\end{array}$ & $\begin{array}{c}\text { Gross } \\
\text { head } \\
\text { weight } \\
(\mathrm{g})\end{array}$ & $\begin{array}{l}\text { Net head } \\
\text { weight } \\
\text { (g) }\end{array}$ & $\begin{array}{c}\text { Polar } \\
\text { diameter } \\
(\mathrm{cm})\end{array}$ & $\begin{array}{l}\text { Equatorial } \\
\text { diameter } \\
(\mathrm{cm})\end{array}$ & $\begin{array}{l}\text { Days to } \\
\text { harvest }\end{array}$ & $\begin{array}{l}\text { Head } \\
\text { shape } \\
\text { index }\end{array}$ & $\begin{array}{c}\text { Compac } \\
\text { tness of } \\
\text { head } \\
\left(\mathrm{g} / \mathrm{cm}^{3}\right)\end{array}$ & $\begin{array}{c}\text { Marketa } \\
\text { ble heads } \\
\text { per plot }\end{array}$ & $\begin{array}{l}\text { Ascorbic } \\
\text { acid (mg/ } \\
100 \mathrm{~g})\end{array}$ & TSS & $\begin{array}{c}\text { Marketa } \\
\text { ble head } \\
\text { yield } \\
\text { (kg/plot) }\end{array}$ \\
\hline \multirow{2}{*}{$\begin{array}{l}\text { Plant } \\
\text { spread }(\mathrm{cm})\end{array}$} & $\mathrm{P}$ & $0.359^{* *}$ & $0.520^{* *}$ & $0.469^{* *}$ & $0.435^{* *}$ & $0.464^{* *}$ & -0.134 & -0.133 & $0.194^{*}$ & 0.154 & 0.044 & 0.156 & $0.427^{* *}$ \\
\hline & G & $0.478^{* *}$ & $0.574^{* *}$ & $0.524^{* *}$ & $0.651^{* *}$ & $0.721^{* *}$ & -0.166 & $-0.424^{* *}$ & $0.277^{* *}$ & $0.222^{*}$ & 0.043 & 0.184 & $0.462^{* *}$ \\
\hline \multirow{2}{*}{$\begin{array}{l}\text { No of non } \\
\text { wrapper } \\
\text { leaves }\end{array}$} & $\mathrm{P}$ & & $0.259^{* *}$ & 0.114 & 0.137 & 0.182 & 0.043 & -0.092 & -0.082 & -0.007 & -0.062 & -0.123 & 0.090 \\
\hline & G & & $0.361^{* *}$ & $0.209^{*}$ & $0.420^{* *}$ & $0.419^{* *}$ & 0.013 & $-0.189^{*}$ & -0.167 & 0.121 & -0.062 & -0.137 & $0.190^{*}$ \\
\hline \multirow{2}{*}{$\begin{array}{l}\text { Gross head } \\
\text { weight }(\mathrm{g}) \\
\end{array}$} & $\mathrm{P}$ & & & $0.891^{* *}$ & $0.548^{* *}$ & $0.672^{* *}$ & $-0.404^{* *}$ & $-0.254^{* *}$ & $0.270^{* *}$ & $0.352^{* *}$ & 0.165 & 0.119 & $0.833^{* *}$ \\
\hline & G & & & $0.950^{* *}$ & $0.887^{* *}$ & $0.973^{* *}$ & $-0.531^{* *}$ & $-0.434^{* *}$ & $0.354^{* *}$ & $0.586^{* *}$ & $0.224^{*}$ & 0.144 & $0.902^{* *}$ \\
\hline \multirow{2}{*}{$\begin{array}{l}\text { Net head } \\
\text { weight }(g)\end{array}$} & $\mathrm{P}$ & & & & $0.577^{* * *}$ & $0.635^{* *}$ & $-0.490^{* * *}$ & -0.172 & $0.315^{* * *}$ & $0.379^{* *}$ & $0.225^{*}$ & 0.176 & $0.940^{* *}$ \\
\hline & G & & & & $0.833^{* *}$ & $0.871^{* *}$ & $-0.644^{* *}$ & $-0.373^{* *}$ & $0.507^{* *}$ & $0.669^{* *}$ & $0.267^{* *}$ & $0.234^{*}$ & $0.982^{* *}$ \\
\hline \multirow{2}{*}{$\begin{array}{l}\text { Polar } \\
\text { diameter }(\mathbf{c m})\end{array}$} & $\mathrm{P}$ & & & & & $0.635^{* *}$ & $-0.254^{* *}$ & $0.231^{*}$ & -0.047 & $0.256^{* *}$ & 0.174 & 0.108 & $0.538^{* *}$ \\
\hline & G & & & & & $0.874^{* *}$ & $-0.536^{* *}$ & $-0.185^{*}$ & $0.382^{* *}$ & $0.597^{* *}$ & 0.146 & 0.169 & $0.799^{* *}$ \\
\hline \multirow{2}{*}{$\begin{array}{l}\text { Equatorial } \\
\text { diameter (cm) }\end{array}$} & $\mathrm{P}$ & & & & & & $-0.340^{* *}$ & $-0.564^{* *}$ & 0.063 & $0.415^{* *}$ & 0.147 & $0.192^{*}$ & $0.651^{* *}$ \\
\hline & $\mathrm{G}$ & & & & & & $-0.633^{* *}$ & $-0.621^{* *}$ & $0.455^{* *}$ & $0.754^{* *}$ & $0.305^{* *}$ & $0.292^{* *}$ & $0.866^{* *}$ \\
\hline \multirow{2}{*}{$\begin{array}{l}\text { Days to } \\
\text { harvest }\end{array}$} & $\mathrm{P}$ & & & & & & & 0.172 & $-0.199^{*}$ & $-0.483^{* *}$ & -0.119 & $-0.220^{*}$ & $-0.553^{* *}$ \\
\hline & $\mathrm{G}$ & & & & & & & $0.447^{* *}$ & $-0.312^{* *}$ & $-0.842^{* *}$ & $-0.220^{*}$ & $-0.281^{* *}$ & $-0.712^{* *}$ \\
\hline \multirow{2}{*}{$\begin{array}{l}\text { Head shape } \\
\text { index }\end{array}$} & $\mathrm{P}$ & & & & & & & & -0.164 & $-0.301^{* *}$ & 0.057 & -0.150 & $-0.255^{* *}$ \\
\hline & $\mathrm{G}$ & & & & & & & & $-0.244^{* *}$ & $-0.551^{* *}$ & $-0.223^{*}$ & $-0.297^{* *}$ & $-0.424^{* *}$ \\
\hline Head & $\mathrm{P}$ & & & & & & & & & $0.285^{* *}$ & 0.068 & 0.149 & $0.397^{* *}$ \\
\hline $\begin{array}{l}\text { Compactness } \\
\left(\mathrm{g} / \mathrm{cm}^{3}\right)\end{array}$ & G & & & & & & & & & $0.526^{* *}$ & $0.264^{* *}$ & $0.233^{*}$ & $0.600^{* *}$ \\
\hline \multirow{2}{*}{$\begin{array}{l}\text { Marketable } \\
\text { heads per plot }\end{array}$} & $\mathrm{P}$ & & & & & & & & & & $0.220^{*}$ & $0.312^{* *}$ & $0.647^{* *}$ \\
\hline & G & & & & & & & & & & $0.446^{* *}$ & $0.347^{* *}$ & $0.776^{* * *}$ \\
\hline \multirow{2}{*}{$\begin{array}{l}\text { Ascorbic acid } \\
(\mathrm{mg} / \mathbf{1 0 0 g})\end{array}$} & $\mathrm{P}$ & & & & & & & & & & & 0.111 & $0.270^{* *}$ \\
\hline & $\mathrm{G}$ & & & & & & & & & & & 0.181 & $0.379^{* *}$ \\
\hline \multirow[t]{2}{*}{ TSS } & $\mathrm{P}$ & & & & & & & & & & & & $0.255^{\text {** }}$ \\
\hline & G & & & & & & & & & & & & $0.278^{* *}$ \\
\hline
\end{tabular}

*Significant at $5 \%$ level of significance $* *$ Significant at $1 \%$ level of significance

Table.2 Direct and indirect effects of component traits on marketable yield of cabbage at phenotypic and genotypic level 


\begin{tabular}{|c|c|c|c|c|c|c|c|c|c|c|c|c|c|c|}
\hline \multicolumn{2}{|l|}{ Traits } & $\begin{array}{l}\text { Plant } \\
\text { spread } \\
(\mathrm{cm})\end{array}$ & $\begin{array}{c}\text { No of } \\
\text { non } \\
\text { wrapper } \\
\text { leaves }\end{array}$ & $\begin{array}{c}\text { Gross } \\
\text { head } \\
\text { weight } \\
\text { (g) }\end{array}$ & $\begin{array}{c}\text { Net } \\
\text { head } \\
\text { weight } \\
(\mathrm{g})\end{array}$ & $\begin{array}{c}\text { Polar } \\
\text { diameter } \\
(\mathrm{cm})\end{array}$ & $\begin{array}{c}\text { Equatorial } \\
\text { diameter }(\mathbf{c m})\end{array}$ & $\begin{array}{c}\text { Days } \\
\text { to } \\
\text { harvest }\end{array}$ & $\begin{array}{l}\text { Head } \\
\text { shape } \\
\text { index }\end{array}$ & $\begin{array}{c}\text { Head } \\
\text { Compactness } \\
\left(\mathrm{g} / \mathrm{cm}^{3}\right)\end{array}$ & $\begin{array}{c}\text { Marketable } \\
\text { heads per } \\
\text { plot }\end{array}$ & $\begin{array}{l}\text { Ascorbic } \\
\text { acid } \\
\text { content } \\
(\mathrm{mg} / 100 \mathrm{~g})\end{array}$ & TSS & $\begin{array}{c}\text { Correlation } \\
\text { with } \\
\text { marketable } \\
\text { yield } \\
\end{array}$ \\
\hline \multirow[t]{2}{*}{ Plant spread(cm) } & $\mathrm{P}$ & $\underline{-0.014}$ & 0.006 & -0.028 & 0.398 & 0.004 & -0.003 & -0.002 & 0.003 & 0.010 & 0.050 & 0.001 & 0.001 & $0.427^{* *}$ \\
\hline & $\mathrm{G}$ & $\underline{-0.134}$ & 0.054 & -0.100 & 0.517 & 0.148 & -0.149 & 0.016 & 0.075 & 0.041 & -0.015 & 0.005 & 0.004 & $0.462^{* *}$ \\
\hline \multirow{2}{*}{$\begin{array}{l}\text { No of non } \\
\text { wrapper leaves } \\
\end{array}$} & $\mathrm{P}$ & -0.005 & $\underline{0.017}$ & -0.014 & 0.097 & 0.001 & -0.001 & 0.001 & 0.002 & -0.004 & -0.002 & -0.001 & -0.001 & 0.090 \\
\hline & $\mathrm{G}$ & -0.064 & $\overline{\mathbf{0 . 1 1 3}}$ & -0.063 & 0.206 & 0.095 & -0.087 & -0.001 & 0.033 & -0.025 & -0.008 & -0.008 & -0.003 & $0.190^{*}$ \\
\hline \multirow{2}{*}{$\begin{array}{l}\text { Gross head } \\
\text { weight }(\mathrm{g})\end{array}$} & $\mathrm{P}$ & -0.007 & 0.004 & $\underline{-0.054}$ & 0.756 & 0.006 & -0.004 & -0.006 & 0.006 & 0.014 & 0.115 & 0.003 & 0.000 & $0.833^{* *}$ \\
\hline & $\mathrm{G}$ & -0.077 & 0.041 & $\underline{-0.174}$ & 0.939 & 0.201 & -0.201 & 0.053 & 0.076 & 0.052 & -0.039 & 0.027 & 0.003 & $0.902 * *$ \\
\hline \multirow{2}{*}{$\begin{array}{l}\text { Net head } \\
\text { weight }(\mathrm{g}) \\
\end{array}$} & $\mathrm{P}$ & -0.006 & 0.002 & -0.048 & $\underline{0.849}$ & 0.006 & -0.004 & -0.007 & 0.004 & 0.017 & 0.124 & 0.004 & 0.001 & $0.940^{* *}$ \\
\hline & $\mathrm{G}$ & -0.070 & 0.024 & -0.165 & $\underline{0.988}$ & 0.189 & -0.180 & 0.064 & 0.066 & 0.075 & -0.044 & 0.033 & 0.005 & $0.982^{* *}$ \\
\hline \multirow{2}{*}{$\begin{array}{l}\text { Polar } \\
\text { diameter }(\mathbf{c m})\end{array}$} & $\mathrm{P}$ & -0.006 & 0.002 & -0.030 & 0.489 & $\underline{0.010}$ & -0.004 & -0.004 & -0.006 & -0.003 & 0.084 & 0.003 & 0.000 & $0.538^{* *}$ \\
\hline & G & -0.087 & 0.047 & -0.154 & 0.823 & 0.227 & -0.181 & 0.053 & 0.033 & 0.057 & -0.040 & 0.018 & 0.003 & $0.799^{* *}$ \\
\hline \multirow{2}{*}{$\begin{array}{l}\text { Equatorial } \\
\text { diameter }(\mathrm{cm})\end{array}$} & $\mathrm{P}$ & -0.006 & 0.003 & -0.036 & 0.539 & 0.007 & $\underline{-0.006}$ & -0.005 & 0.014 & 0.003 & 0.136 & 0.002 & 0.001 & $0.651^{* *}$ \\
\hline & $\mathrm{G}$ & -0.097 & 0.047 & -0.169 & 0.861 & 0.198 & $\underline{-0.207}$ & 0.063 & 0.110 & 0.067 & -0.050 & 0.037 & 0.006 & $0.866^{* *}$ \\
\hline \multirow[t]{2}{*}{ Days to harvest } & $\mathrm{P}$ & 0.002 & 0.001 & 0.022 & -0.415 & -0.003 & 0.002 & $\underline{0.014}$ & -0.004 & -0.011 & -0.158 & -0.002 & -0.001 & $-0.553 * *$ \\
\hline & G & 0.022 & 0.001 & 0.092 & -0.637 & -0.122 & 0.131 & $\underline{-0.099}$ & -0.079 & -0.046 & 0.056 & -0.027 & -0.006 & $-0.712 * *$ \\
\hline \multirow{2}{*}{$\begin{array}{l}\text { Head shape } \\
\text { index }\end{array}$} & $\mathrm{P}$ & 0.002 & -0.002 & 0.014 & -0.146 & 0.002 & 0.003 & 0.002 & $\underline{-0.024}$ & -0.009 & -0.098 & 0.001 & -0.001 & $-0.255^{* *}$ \\
\hline & G & 0.057 & -0.021 & 0.075 & -0.368 & -0.042 & 0.129 & -0.044 & $\underline{-0.176}$ & -0.036 & 0.037 & -0.027 & -0.006 & $-0.424 * *$ \\
\hline \multirow{2}{*}{$\begin{array}{l}\text { Head } \\
\text { Compactness } \\
\left(\mathrm{g} / \mathrm{cm}^{3}\right)\end{array}$} & $\mathrm{P}$ & -0.003 & -0.001 & -0.015 & 0.267 & 0.000 & 0.000 & -0.003 & 0.004 & $\underline{0.053}$ & 0.093 & 0.001 & 0.001 & $0.397 * *$ \\
\hline & G & -0.037 & -0.019 & -0.062 & 0.501 & 0.087 & -0.094 & 0.031 & 0.043 & $\overline{0.148}$ & -0.035 & 0.032 & 0.005 & $0.600^{* *}$ \\
\hline \multirow{2}{*}{$\begin{array}{l}\text { Marketable } \\
\text { heads per plot }\end{array}$} & $\mathrm{P}$ & -0.002 & 0.000 & -0.019 & 0.321 & 0.003 & -0.002 & -0.007 & 0.007 & 0.015 & $\underline{0.327}$ & 0.004 & 0.001 & $0.647^{* * *}$ \\
\hline & G & -0.030 & 0.014 & -0.102 & 0.661 & 0.135 & -0.156 & 0.084 & 0.097 & 0.078 & $\underline{-0.066}$ & 0.055 & 0.007 & $0.776^{* *}$ \\
\hline \multirow{2}{*}{$\begin{array}{l}\text { Ascorbic acid } \\
\text { content(mg/100g) }\end{array}$} & $\mathrm{P}$ & -0.001 & -0.001 & -0.009 & 0.191 & 0.002 & -0.001 & -0.002 & -0.001 & 0.004 & 0.072 & $\underline{0.016}$ & 0.000 & $0.270^{* *}$ \\
\hline & $\mathrm{G}$ & -0.006 & -0.007 & -0.039 & 0.264 & 0.033 & -0.063 & 0.022 & 0.039 & 0.039 & -0.030 & $\underline{0.123}$ & 0.004 & $0.379^{* *}$ \\
\hline \multirow[t]{2}{*}{ TSS } & $\mathrm{P}$ & -0.002 & -0.002 & -0.006 & 0.149 & 0.001 & -0.001 & -0.003 & 0.004 & 0.008 & 0.102 & 0.002 & $\underline{0.004}$ & $0.255^{* *}$ \\
\hline & $\mathrm{G}$ & -0.025 & -0.016 & -0.025 & 0.231 & 0.038 & -0.060 & 0.028 & 0.052 & 0.034 & -0.023 & 0.022 & $\underline{0.020}$ & $0.278^{* *}$ \\
\hline
\end{tabular}

** Significant at $\mathrm{P} \leq 0.01$; *Significant at $\mathrm{P} \leq 0.05$ level; Residual effect $(\mathrm{P}): 0.014 ;(\mathrm{G})$ : -0.098 ; the bold values indicate direct effect with marketable yield per plot 
Earlier researchers also reported positive direct effects of net head weight (Singh et al., 2010, Sharma, 2010 and Soni et al., 2013), marketable heads (Sharma, 2010). Direct negative effects were reported for gross head weight by Sharma (2010). Direct positive effects were observed for gross head weight by Meena et al., (2010) and Singh et al., (2010).

At phenotypic level, the maximum positive indirect effects on marketable yield per plot were observed by gross head weight (0.756) followed by equatorial diameter (0.539), polar diameter (0.489), plant spread (0.398), marketable heads per plot (0.321) and compactness of head (0.267) via net head weight. Therefore the net head weight is the single most important character which is required to be considered to improve upon marketable head yield and other horticultural traits. The results are in consonance with the findings of Sharma (2010) and Soni et al., 2013.

\section{Acknowledgements}

Authors are highly thankful to ICAR for providing funds under Consortium Research Platform project on hybrid development in cabbage.

\section{References}

Al-Jibouri, H.A., P.A, Miller and Robinson, H.F. 1958. Genotypic and environmental variances and covariances in an upland cotton cross of interspecific origin. Agronomy Journal. 50: 633-636.

Anonymous. 2016. National Horticultural Board, Gurgaon (Haryana). www.nhb.gov.in

Anonymous. 2015. Area, production and productivity of cabbage in Himachal Pradesh. Department of Agriculture
(H.P) Shimla.

Burton G.W., and De Vane E.H. 1953. Estimating heritability in tall fescue (Festuca arundinacea) from replicated clonal material. Agronomy Journal. 45: 478-48.

Dewey D.R., and Lu K.H. 1959. A correlation and path coefficient analysis of components of crested wheat grass seed population. Agronomy Journal. 51: 515518.

Kibar, B., O. Karaagac and Kar, H. 2014. Correlation and path coefficient analysis of yield components in cabbage (Brassica oleracea var. capitata L.). Acta Scientiarum Polonorum., Hortorum Cultus. 13: 87-97.

Kopsell, D.A., D.E. Kopsell and Lefsrud, M.G. 2004. Variation in lutein, Bcarotene and chlorophyll concentrations among leafy Brassica oleracea cultigens and seasons. Horticulture Science 39: 361-364.

Kumar, C., H.C. Raturi and Uniyal, S.P. 2013. Performance of cabbage hybrids under rainfed mid hills conditions of Uttrakhand. Asian Journal of Horticulture 8(1): 110-113.

Meena, M.L., R.B. Ram and Rubee, L. 2009. Genetic variability and correlation studies for some quantitative traits in cabbage (Brassica oleracea var. capitata L.) under Lucknow conditions. Progressive Horticulture. 41: 89-93.

Sharma, K.C. 2010. Genetic variability, characters association and path analysis in exotic lines of cabbage under mid hill, sub-humid conditions of Himachal Pradesh. Journal of Hill Agriculture. 1: 146-150.

Sharma, P. 1994. Genetic variability and path analysis in cabbage (Brassica oleracea var. capitata L.), M. Sc. Thesis, 59p. Department of Vegetable Science and Floriculture, CSK Himachal Pradesh Krishi Vishvavidyalaya, Palampur, 
Himachal Pradesh, India.

Singh, B., A.K. Mishra, S.K. Sanwal, P.K. Singh and Rai, M. 2013. Genetic variability and character association analysis in cabbage hybrids. Indian Journal of Horticulture. 70: 296-299.

Singh, B.K., S.R. Sharma and Singh, B. 2009. Heterosis for mineral elements in single cross-hybrids of cabbage (Brassica oleracea var. capitata L.). Scientia Horticulturae. 122: 32-36.

Singh, B.K., S.R. Sharma, P. Kalia and Singh,
B. 2010. Character association and path analysis of morphological and economic traits in cabbage (Brassica oleracea var. capitata L.). Indian Journal of Agriculture Sciences. 80: 116-118.

Soni, S., S. Kumar and Maji, S. 2013. Correlation and path coefficient analysis studies for the yield and its traits in cabbage (Brassica oleracea var. capitata L.). Annals of Horticulture. 6: 331-336.

\section{How to cite this article:}

Manmeet Kaur, Sanjay Chadha, Nitish Kumar, Nidhi Sehgal and Sangeeta Kanwar. 2018. Characters Association and Path Analysis among CMS and SI Based Cabbage Hybrids under Mid Hill Conditions of Himachal Pradesh, India. Int.J.Curr.Microbiol.App.Sci. 7(01): 424-430. doi: https://doi.org/10.20546/ijcmas.2018.701.049 